\title{
AUTOMATIC KNEE SEGMENTATION USING EAGLE ALGORITHM WITH MULTI STOCHASTIC OBJECTIVE PROCESS
}

G. Rini

Research Scholar, Department of EGE, Kalasalingam Academy of Research and Education.

Krishnankoil, Virudhunagar (Dt), (India).

E-mail: rinidass2002@yahoo.co.in

ORCID: https://orcid.org/0000-0002-4413-5850

B. Perumal

Associate Professor, Department of ECE, Kalasalingam Academy of Research and Education. Krishnankoil, Virudhunagar (Dt), (India).

E-mail: palanimet@gmail.com

ORCID: https:// orcid.org/0000-0003-4408-9396

M. Pallikonda Rajasekaran

Professor, Department of ECE, Kalasalingam Academy of Research and Education.

Krishnankoil, Virudhunagar (Dt), (India).

E-mail:mpraja80@gmail.com

ORCID: https://orcid.org/0000-0001-6942-4512

V. Muneeswaran

Assistant Professor, Department of ECE, Kalasalingam Academy of Research and Education. Krishnankoil, Virudhunagar (Dt), (India).

E-mail: munees.klu@gmail.com

ORCID: https://orcid.org/0000-0001-8061-8529

Recepción: 11/11/2019 Aceptación: 21/12/2020 Publicación: 30/11/2021

\section{Citación sugerida:}

Rini, C., Perumal, B., Rajasekaran, M. P., y Muneeswaran, V. (2021). Automatic knee segmentation using eagle algorithm with multi stochastic objective process. 3C Tecnología. Glosas de innovación aplicadas a la pyme, Edición Especial, (noviembre, 2021), 333-353. https://doi.org/10.17993/3ctecno.2021. specialissue8.333-353 


\section{ABSTRACT}

In our living world, Osteoarthritis $(\mathrm{OA})$ is known to be foremost sicknesses which affect the knee region especially affect hoarier people. The reason for OA in a person may be due to ageing, malformed joints, rough cartilage, genetics effects or continuous repetitive stress towards the joint. Magnetic Resonance Imaging (MRI) enacts a vigorous role in the medical field for detecting issues regarding bone structure, cartilage, and meniscus region and tibia bone. Though it provides the details about the bone, it is not useful to detect clearly about the faults in the bone due to many unfavorable conditions like poor segmentation, broken pixels or some other natural issues including the shakes while clicking the image, blurring, etc. Besides, manual calculations have some unexceptional error with partial accuracy. Hence automated segmentation should be implemented for achieving perfection in accuracy and the bone segmentation. In our work, we proposed eagle algorithm as the segmentation method which provides an improved accuracy in contrast with other traditional methods. The performance is measured by the metrics such as thickness, mean and Standard Deviation (SD).

\section{KEYWORDS}

Pre-Processing, Contrast Enhancement, Stochastic Multi-Objective Process, Levy Walk Random Process, Eagle Algorithm. 


\section{INTRODUCTION}

Generally, 3-D Magnetic Resonance (MR) imaging delegates the therapies regarding noninvasive, high-resolution as well as isotropic voxels. On behalf of quantitative studies including dimensional (3-D) reconstruction and dimension of the cartilage, truthful segmentation of the images is obligatory. The main objective of our work is to improve and authenticate software for automated segmentation and thickness representing of articular cartilage from three-dimensional (3-D) gradient-echo MR images of the knee. Inelegantly, random noise occurs throughout the MR image processing and hence the quality of the image is vitiated. Besides, the medical diagnostic tasks and noise damage many image processing and investigation charges like registration, segmentation, super resolution and visualization, etc. Hence the suppression of noise is essential to recover the image quality. Many methods and algorithms are being introduced for attaining clear segmentations are discussed in the next section.

Classically, joints are the regions where two bones meet in our body. Habitually, 360 joints are there in our human body which include 86 skull joints, six throat joints, 66 thorax joints and 76 in the spine and pelvis region. In case of limbs, 32 joints found in each upper limb and 31 joints in each lower limb. Among that, knee joint is solitary and it connect the leg and thigh of our human body. The knee joint is a hinge type synovial joint provide the flexion and extension of the leg. Knee joint is formed by articulations among patella bone, femur bone (thigh bone) and tibia bone (leg bone). There are two articulations in the knee joint. One of them is located between the femur and tibia bone and the latter is built between the patella and femur (Peterfy et al., 2008); Eckstein, Burstein, \& Link, 2006).

On the other hand, Condyle is defined as the round prominence at the end of a bone, a part of the joint which articulate with another bone. It is also said to be one of the markings or features of bones. In the femur bone of the knee joint, condyles are two types of condyles namely medial condyle and lateral condyle. The joints are shaped by means of the condyles of femur bone and tibia bone. The interior structure of knee joint comprise of synovial cavity covering synovial fluid, articular cartilage, meniscus region (semilunar cartilages), cruciate ligaments and burse. Articular cartilage is a silky, white tissue that wrap the ends of bones together with bones to form joints. In the mechanism of joints, 
if the cartilage is fine, it is easier to move. It certifies the bones to glide over each other with a very slight friction.

Articular cartilage which covers patella bone, tibia bone and femur bone are frequently injured by wounds or normal wear and tear. When the thickness of the cartilage is diminished, the growth of Osteoarthritis $(\mathrm{OA})$ begins. Osteoarthritis $(\mathrm{OA})$ is the most common form of arthritis, affect millions of people in worldwide. It occurs when the protective cartilage on the ends of the bones wears down over time. Osteoarthritis symptoms can be effectively managed, although the underlying process cannot be reversed. Maintenance of vigorous weight and other treatments may leads for slow progression of the disease and help recover pain and joint function. The cartilage joint is completely vanished whoever suffering from severe OA.

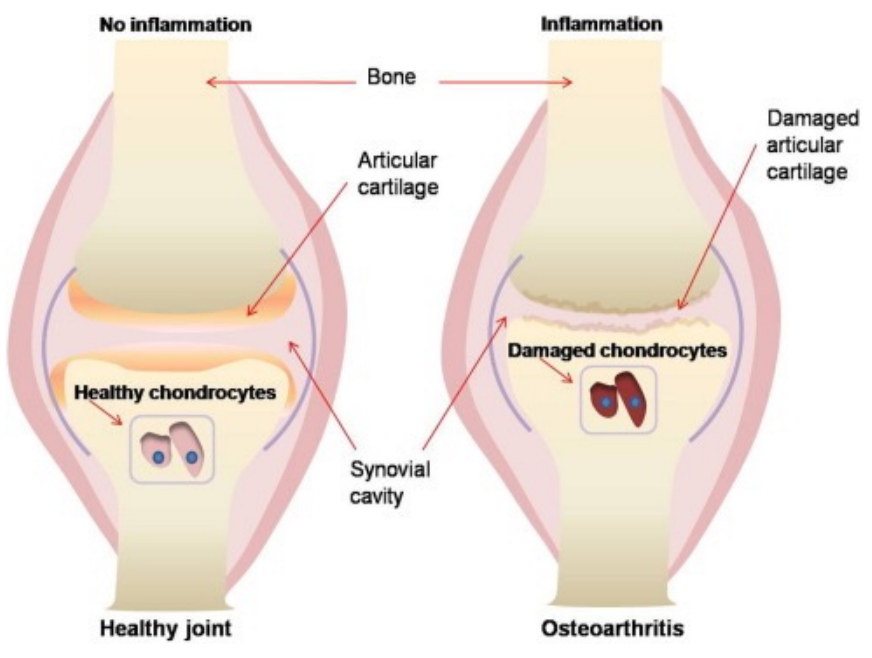

Figure 1. Knee joint for healthy and affected people.

Source: own elaboration.

During the cartilage joint reduction, the synovial fluid drops its lubrication ability which results in pain, inflammation and eased movement of the joint. The bones get scratch while gliding without fluid and new tiny bones called spurs start to develop. Therefore, the cartilage function (shock absorber) is constrained due to the stress on joint result in severe influence. The growth of Osteoarthritis (OA) may be stopped or diminished by introducing a vigorous biomarkers to resolve the issue. An organization named Osteoarthritis Initiative (OAI) has the main objective to detect and analyze the risk factors of Osteoarthritis which 
are performing by several private health organizations. According to their survey, 50.2\% of the people among the age 65 and 74 years and $97.7 \%$ of people of age eighty-four and above, are being affected by this disease.

Body mass Index (BMI) should be maintained for reduction of Osteoarthritis (OA). People with high BMI rage falls under the respected disease and leads to give heavy burden at the joint. The normal value of BMI range for both male and female is 18.5 - 25. People having BMI beyond this range is considered to be overweight and they have the chances to suffer from Osteoarthritis (OA). It was 51.36\% for the people with a BMI below 25 and 100\% for the people with a BMI above 40. The cartilage damage in the joints of knee can be observed by means of Magnetic Resonance Images (MRI). MRI scan is used to scrutinize the in-vivo and in-vitro structures of the human body meanwhile it is non-invasive and has great resolution soft tissue contrast. Sagittal, coronal and axial planes are three different planes where MRI scan the joints. The knee joint MRI images without Osteoarthritis (OA) as publicized in Figure 2(a). At the same time, Figure 2(b) shows the MRI images regarding knee joint of an OA affected person. With numerous time series of MR images, the physicians detect femur, tibia and cartilage of knee bones and observe the influences of OA.

Magnetic Resonance Imaging (MRI) create the magnetic properties of assured atomic nuclei. The human body is mostly covered by water. The hydrogen nuclei present in the water act as compass needles which are incompletely aligned by a strong magnetic field in the scanner. Radio waves rotates the nuclei and oscillate in the magnetic field while returning to equilibrium successively. For examining the tissues, the waves concurrently emit radio signals which are detected by antennas. MRI does not have process like radioactivity or ionization comparing to the traditional methods. The range of Radio Frequency (RF) for the operation is normal and won't affect the body. The signal of MRI is sensitive to a wide range of effects like nuclear mobility, molecular structure, flow and diffusion. 


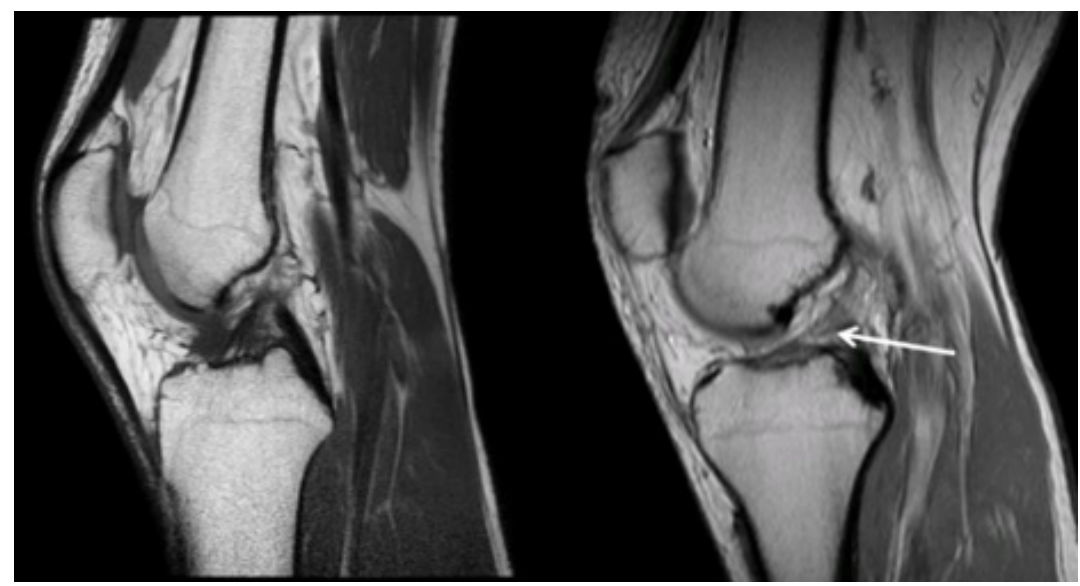

Figure 2. a) MRI of normal knee, b) MRI of OA knee.

Source: own elaboration.

As a consequence, MRI is a very flexible technique that provides the interior results of both structure and function. Initially, the output image of MRI is processed with quantization. In case of knee joints, MRI images are captured for detecting obvious output regarding meniscal lesions, cartilage thickness, reshaping the bones, osteophytes, bone marrow lesions and synovitis (Pelletier et al., 2008). The image segmentation is required to observe the exact issue happened on the knee whereas the manual calculations have partial accuracy. Hence, some auto segmentation methods are introduced for detecting and computing the structure of the bone clearly. In our work, we proposed eagle algorithm (Yang \& Deb, 2010) for segmentation process which executes accurate image quality and reduced pixel breakdown. When compare with the existing method, the accuracy is greatly enhanced and the thickness can be measured obviously.

\section{RELATED WORKS}

Early quantitative MRI studies assured promising morphological cartilage metrics for describing the status of disease and perhaps to screen its progression (Norman, Pedoia, \& Majumdar, 2018). Nonetheless, the recent studies utilize larger OA cohorts by means of demonstrating MRI articular cartilage biomarkers may have limited approachability to disease progression. On other hand, the study has sustained to grow novel and better image segmentation tools (Kubassova, Boyle, \& Pyatnizkiy, 2005; Yin et al., 2010). In the present 
decades, most quantitative analysis methods require uninterrupted human involvement and also should be proficient for accurate cartilage segmentation (Kauffmann et al., 2003). Image acquisition and denoising are the two techniques used to reduce the noise from the images. Average of number of the similar samples that obtained is the result of image acquisition technique. Conventionally, the image is denoised by denoising techniques which acquire less time for computation (Liu et al., 2010).

Novel segmentation algorithm (Peterfy, Schneider, \& Nevitt, 2008) presented knee cartilages with level-based segmentation method and new template data. It gives consequence of three cartilage tissues such as tibia, femur and fibula bone. The segmentation of cartilage is grim in atlas-based segmentation (Pelletier et al., 2008), since cartilage intensity varies by thin or fat and the boundary of the cartilages are girdled by the muscle tissues. A main shortcoming of atlas-based approach is the large inconsistency of the articular cartilage. The potential for preference is equivalent to the target delay in accuracy (Raynauld et al., 2004).

The multi-atlas based algorithm (Pavlyukevich, 2007) overcome atlas based algorithm with promising results where it combine simple label fusion approach but not utilize any other correction methods to report abnormalities like osteophytes produced besides the joint margins. Alternatively, Local Weighted Vote (LWV) algorithm is built where the multi atlas data merges and provide the result (Rini et al., 2020). The segmentation structure grips three procedures including multiple-atlas building, applying a locally weighted vote (LWV) and adjusting the region. In case of atlas building process, every training cases are recorded to a target image - a non-rigid registration method and the finest coordinated atlases are designated. However, LWV algorithm was usually applied for integrating the data from these atlases and produce the initial segmentation outcome. At last, in the region adjustment procedure, the statistical data of bone, cartilage, and surrounding regions is calculated from the preliminary segmentation result. The statistical data absorbed the automated determination of the seed points in all regions of bone intended for the graphcut based method.

The locus-correlated background by voxel subsampling technique is preferred than uniform or Gaussian subsampling to distinguish the objects of interest from supplementary 
objects. These supplementary objects have similar and close structures that frequently knock into MR images, particularly for objects focusing on highly curved and complex shapes (Stammberger et al., 1999). In this approach, cartilage compartments are segmented underprivileged of prior segmentation of bones or determination of Bone Cartilage Interface (BCI) demanding multiple MR images of a participant. Recently, a novel method is introduced called U-net model whose results show longitudinal precision proves the heftiness of algorithm, free of the ground truth definition (Norman et al., 2018).

The recent convolutional neural networks produce fast, accurate, and precise automatic segmentations in cartilage and meniscus compartments which are invariant across patients with OA. This approach also have continuous compensation of computational speed and established efficacy in extracting relaxation times and morphologic features which used in the prediction and intensive care of joint degeneration in OA. Local Coordinate System (LCS) is a method used to segment the femoral and tibia cartilage regions based on 2-D active contour algorithm (Kauffmann et al., 2003). This method obtains a uniform sketch of cartilage dimensions. Cartilage regions were usually segmented in the 3D-MRI scan and developed into offset maps with certain gaps acquired from an inter-slice distance. The offset maps were expressed by Local Coordinate System (LCS).

The gaps are filled with bi-cubic interpolation process. Several segmentation methods of knee joints are performed on semi-automatic segmentation such as B-spline snakes (Stammberger et al., 1999), active contours (Lynch et al., 2000; Raynauld et al., 2004), directional gradient vector flow snakes (Tang et al., 2006), region-growing scheme (Stammberger et al., 1999) and extended watershed algorithm, where others are achieved habitually akin to voxel classification algorithm (Stammberger et al., 1999) and statistical shape model (Fripp et al., 2007). These approaches explain cartilage boundaries in twoor three-dimensional image spaces. Owing to the curved and thin structure of cartilage in core, the cartilage segmentation job are quite complex in stimulation. In the work of Grau et al. (2004), it does not permit informal control of segmentation outflow or objective tessellation but suffer with lack of stability and excessive sensitivity to noise.

The reported techniques used basic signal analysis such as directional edge filters (Wolf, Weierich, \& Niemann, 1997), mathematical morphology (Dogdas, Shattuck, \& Leahy, 
2002), gray-level classification (Folkesson et al., 2005), histogram analysis (Kubassova, Boyle, \& Pyatnizkiy, 2005), and other techniques including the hybrid signal and model, e.g., texture level-set (Lorigo et al., 1998) and model fitting. Nonetheless, these solutions needed a specific tuning which is responsible for the mutable image quality or the signal corrosion owing to disease. This tuning trusts on initial manual contribution, additional signal data or cooperating tuning by making the independent use of these methods difficult in the context of a large scale process. Alternatively, an algebraic strategy called DouglasRachford Splitting method is presented in the work of Rini, Perumal, and Rajasekaran (2020) which is segmented by means of proximal splitting method. Various applications of image processing have been demonstrated in the literature (Muneeswaran \& Rajasekaran, 2016, 2017, 2019c).

\section{MATERIALS AND METHODS}

The block diagram of the proposed Eagle Algorithm (EA) method is shown in Figure 3. The input image is the MRI images of the Osteoarthritis (OA) affected knee image. In case of image processing, image acquisition is mandatory to transform the analogous image into a digitalized form. It is done by the process of sampling and quantization. The spatial resolution of the digitalized image is determined by the sampling rate, whereas the quantization level controls the number of gray levels in the digitized image. An acquisitioned image is send as input to the preprocessing where the contrast and the illumination of the image is completely adjusted. Eagle algorithm extract the thickness of the cartilage region from the femur, tibia or patella bone and segment the portion. The image is then quantized for desired number of outputs. The output image is the noiseless and hassle free MRI image which can detect the thickness of the cartilage precisely.

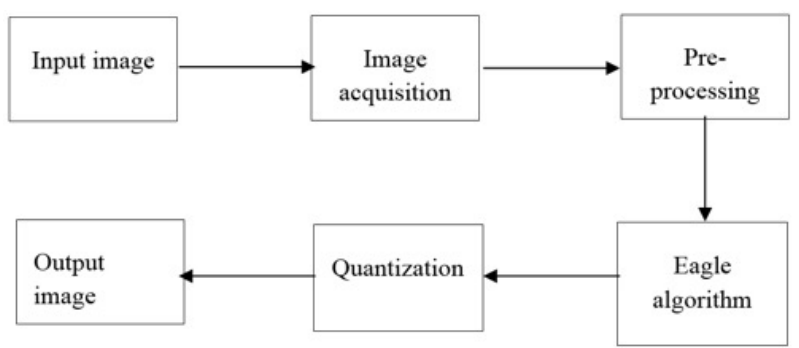

Figure 3. Block diagram of the proposed method.

Source: own elaboration. 


\subsection{PRE-PROCESSING}

The purpose of preprocessing is to convert the RGB image into gray image. The image cannot be processed under the RGB image. Hence it is converted into the binary image (grey scale image). The gray scale image suffer with the issues like contrast, illumination and blur. In our proposed method, these problems are resolved by using Contrast Enhancement Method (CHE). CHE provides perfect lightning effect regarding the background and the Anisotropic Filter (AF) eradicate the noise in image. The Anisotropic Filter (AF) alters the image to concentrate on the extracted portion where the edges are turned to be blurred. This filter is non-linear type that permit dispersion in the consistent area and obstructs at the boundaries. The partial differential equation (PDE) of anisotropic filter is given by Equation (1).

$$
\left\{\begin{array}{l}
\frac{\partial A}{\partial t} \\
A(t=0)=A_{o}
\end{array}=\operatorname{div}[c(|\nabla A|) \nabla A\}\right.
$$

Where $\nabla$ - gradient operator, div - divergence operator, $A_{0}$ - Initial image and $c(|\nabla A|)$ diffusion coefficient, which is represented as

$$
c(x, y)=e\left(-\left[\frac{\nabla A(x, y)}{k}\right]^{2}\right)
$$

The input image (A) is a gray level 3-D image from MRI scanning, where the gray value of a pixel at a point $\left(a^{\prime}, b^{\prime}, c^{\prime}\right)$ is specified by $x=\left(a^{\prime}, b^{\prime}, c^{\prime}\right)$. Thus, the slices of the 3-D image are signified as 2-D images, $A \mathrm{a}$ '.

$$
\begin{aligned}
& A_{a^{\prime}=h} \equiv\left\{A\left(a^{\prime}, b^{\prime}, c^{\prime}\right) /\left(a^{\prime}=h\right)\right\} \\
& A_{b^{\prime}=j} \equiv\left\{A\left(a^{\prime}, b^{\prime}, c^{\prime}\right) /\left(b^{\prime}=j\right)\right\} \\
& A_{c^{\prime}=t} \equiv\left\{A\left(a^{\prime}, b^{\prime}, c^{\prime}\right) /\left(c^{\prime}=t\right)\right\}
\end{aligned}
$$

The 2D images are outlined as 3D image limits having gray levels. The image space $\mathrm{S}$ is the collection of all points of $\left(\mathrm{a}^{\prime}, \mathrm{b}^{\prime}, \mathrm{c}^{\prime}\right)$ and the slices for the groups are $\Omega \mathrm{a}^{\prime}=h=\left\{\left(\mathrm{b}^{\prime}, \mathrm{c}\right\}\right)$ $A \mathrm{a}=h>0\}$, idemfor $\Omega \mathrm{b}^{\prime}=\mathrm{j}, \Omega \mathrm{c}^{\prime}=t$ and these spaces are called as axial, coronal and sagittal slices respectively. The 3-D image $A$ can be defined as a discrete function of the Euclidian 3-D space and their measurements are represented as,

$$
\left(a^{\prime}, b^{\prime}, c^{\prime}\right)=\left(a^{\prime}, b^{\prime}, c^{\prime}\right) \in Z^{3}
$$


For the given bone region $(\phi)$, the femur region or tibia region are in the same MR image $A$, then the region is defined by two factors $(\mathrm{u}, \mathrm{v})$ as given in equation (4),

$$
\left(\mathrm{a}^{\prime}, \mathrm{b}^{\prime}, \mathrm{c}^{\prime}\right)=(u, v)\left(\mathrm{a}^{\prime}, \mathrm{b}^{\prime}, \mathrm{c}^{\prime}\right) \in R^{3}
$$

The bone region $(\phi)$ is a reference region which have a volume of interest in the whole cartilage. The values of $a^{\prime}, b^{\prime}$ and $c^{\prime}$ indices are integer values, since the values of the 3-D image are digital. Similarly a set $D$ is computed by implicating the equation through intensity level $l$ which is discrete.

$$
V=\left\{\left(\mathrm{a}^{\prime}, \mathrm{b}^{\prime}, \mathrm{c}^{\prime}\right) /\left(\mathrm{a}^{\prime}, \mathrm{b}^{\prime}, \mathrm{c}^{\prime}\right)=l\right\}
$$

The voxels is a set of unit cubes in $R^{3}$ which is similar to the coordinate axes and midpoints are located in $Z^{3}$.

\subsection{STOCHASTIC MULTI OBJECTIVE OPTIMIZATION}

Generally, a regular optimization problem without including any noise or uncertainty, can be given by

$$
\begin{gathered}
\min _{x \in R^{d}} f_{i}(s), i(1,2, \ldots, N) \\
\emptyset_{j}(S)=0,(j=1,2,3, \ldots, N) \\
\psi_{k}(S)=0,(k=1,2,3, \ldots, K)
\end{gathered}
$$

Where $\mathrm{s}=(\mathrm{s} 1, \mathrm{~s} 2 \ldots \mathrm{sd})^{\mathrm{T}}$ is the vector of design variables.

Monte Carlo method is a sampling technique to determine $\mu f$. After the samples drawn randomly, we have

$$
\mu_{f_{i}} \approx \frac{1}{M_{i}} \sum_{p=1}^{M_{i}} f_{i}\left(X, \xi^{(p)}\right)
$$

Where $M_{i}$ denotes the number of samples.

\subsection{EAGLE ALGORITHM}

The scavenging behavior of eagles especially the behavior of golden eagle is motivating. Generally, an eagle feeds in its own region by flying freely in a random manner similar to the Levy flights. When the prey is found by eagle, automatically it will alter its search strategy to a concentrated chasing strategies to catch the prey as efficiently as possible. Thus, 
two important mechanisms are involved in an eagle's hunting strategy: random search by Levy flight (or walk) and intensive chase by fastening its aim on the objective. The work of Reynolds and Frye works with fruit flies, see the sights at their landscape by means of a series of straight flight paths interrupted by an unexpected 900 turn, prominent to a Levyflight-style sporadic scale-free search pattern. However, light also can be connected to Levy flights (Barthelemy, Bertolotti, \& Wiersma, 2008). Consequently, this kind of behavior has been utilized in the process of optimization and optimal search, and preliminary results depicted with its enhanced capability (Pavlyukevich, 2007).

In our proposed work, let us fetishize the two-stage approach of an eagle's foraging behavior. At first, assumption is made that an eagle has to do the Levy walk in the entire domain. After the detection of prey, it alters to a chase strategy. Next, the chase strategy can be measured as an intensive local search using any optimization techniques. Likewise, the output image of the Anisotropic Filter (AF) is scanned randomly for the segmentation process. When it detects the area of region where have to be segment, then the area is intensely chased and segmented. Since the area of the region is chasing intensely without considering any other region, the pixel is broken in reduced number. In the initial step, EA is a two-stage strategy rather than a simple iterative method which going to conglomerate a better randomization technique of global search with an intensive and well-organized local search method. Additionally, EA applies Levy walk rather than simple randomization, which resource that the global search space can be reconnoitered proficiently. In fact, the studies show that Levy walk is more efficient than simple random-walk exploration. The Levy walk has a random step length being drawn from a Levy distribution which is given by

$$
\mathrm{EA} \sim \mathrm{v}^{-\lambda},(1<\lambda \leq 3)
$$

Where $\mathrm{v}$ represents the infinite variance with infinite mean. Here the steps of the eagle motion is fundamentally a random walk process by means of a power-law step-length distribution with a substantial tail. The special case $(\lambda=3)$ corresponds to Brownian motion, whereas the case $(\lambda=1)$ has a physical appearance of stochastic excavating, which may be more resourceful to escape being surrounded in local optima. In our proposed work, we use the eagle algorithm to do the local search, since the eagle algorithm was designed to crack multimodal global optimization problems. 


\section{RESULTS}

For our proposed work, we have taken an input set with three regions of knee bone namely femur, tibia and patella region. These three samples are tested with every process of Eagle Algorithm (EA) as follows. Initially, the input images of the knee bone region are the gray scale or RGB image which covers the cartilage region as revealed in Figure 4. The input image is converted to gray scale image under the process of preprocessing. Anisotropic Filter (AF) filters the brightness whereas the Contrast Enhancement Method (CHE) adjust the brightness of the image.
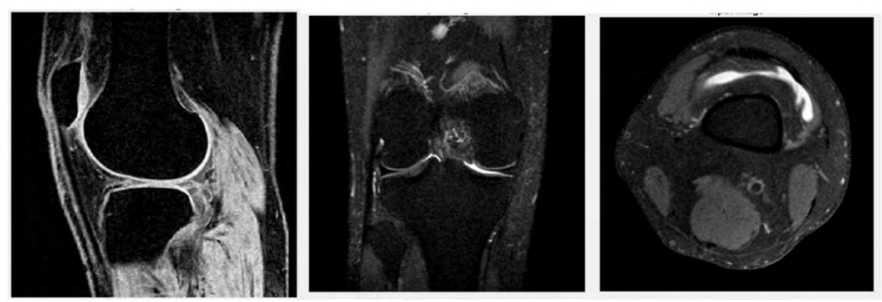

Figure 4. The input images of femur, tibia and patella of knee bone region.

Source: own elaboration.

In case of the binary images, the thresholding process is mandatory. Our technique works with the Otsu method for binarization which further provide contrast enhancement in the binary images. Otsu method produce best results in contrast with the other threshold filters and also ensures the clear view about the thickness of the images as shown in Figure 6.
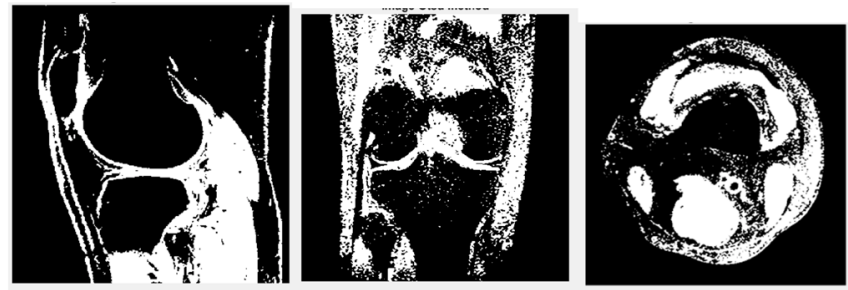

Figure 5. Otsu method output images.

Source: own elaboration.

The contrast enhancement for altering the effect of the color images at the cartilage region as shown in Figure 6. It shown clearly about the cartilage region at where we can obviously view the cartilage region. Comparing the Figure 5 and Figure 6, it is obvious about the optimal differences in the region differentiation. 

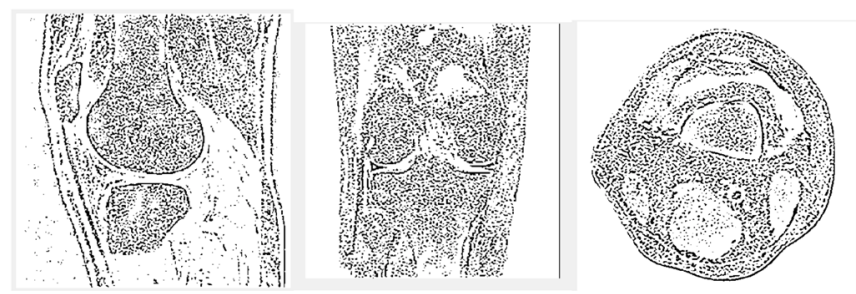

Figure 6. Contrast enhancement.

Source: own elaboration.

The Anisotropic Filter (AF) is included with the process to remove the noise and region of interest (ROI) of the image as shown in Figure 7. From this depicted figure, we can observe the removal of noise at the segmenting region.
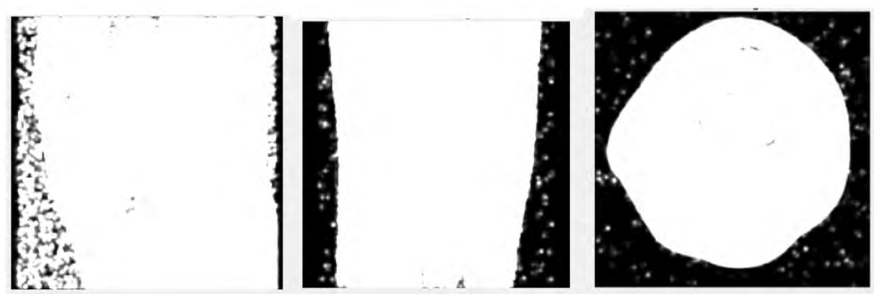

Figure 7. Anisotropic Filter (AF) image.

Source: own elaboration.

As we discussed above, the Eagle Algorithm (EA) is categorized under levy walk process for extracting the area pattern and intensive chasing for identifying the thickness of the pattern. The stochastic multi objective process deliberates the random search to extract that where the thickness has to be determined. After it founds the region, it concentrates on the desired pattern whereas another surround with blur as shown in Figure 8. 


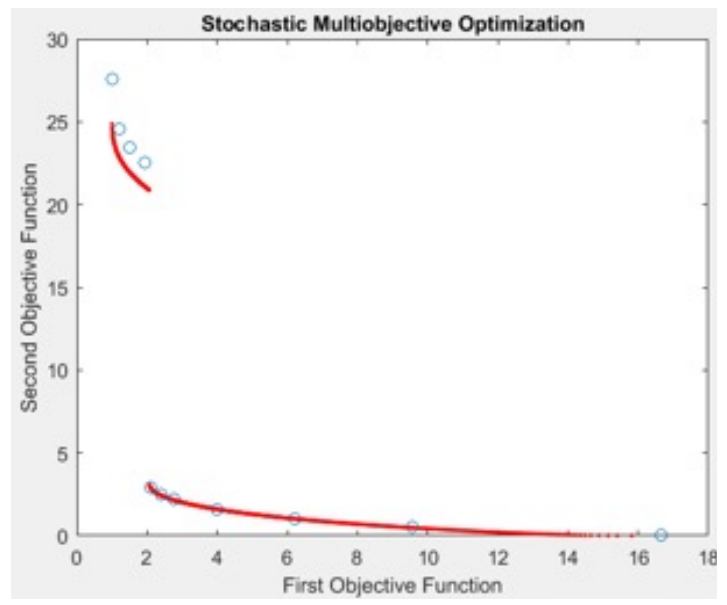

Figure 8. Stochastic multi objective process.

Source: own elaboration.

The above figure illustrates the probability of achieving maximum thickness for several input set images. The objective function of the scale measures the value of the accuracy in the images. It is found that the images are steadily removed by the noise and after the completion of the search. In contrast with the traditional method, less computation time is required in this process. The power law regression of the set of data sets of MRI images are depicted in Figure 9.

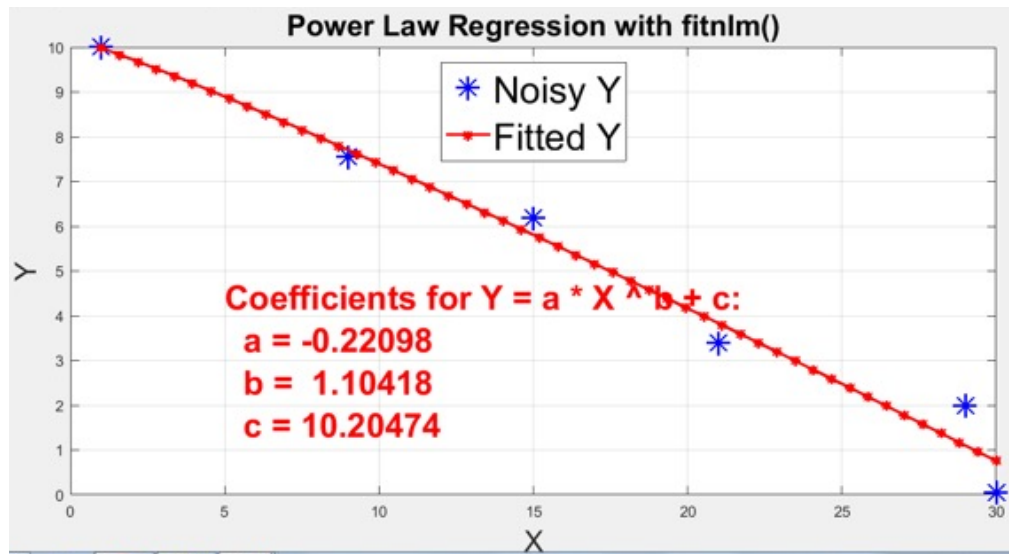

Figure 9. Power law regression.

Source: own elaboration. 


\subsection{PERFORMANCE METRICS}

The quality measurement of the output image is dignified by means of the thickness, mean and the standard deviation as publicized in Table 1 . The proposed work greatly enhanced accuracy of the image when compare with the existing method.

Table 1. Performance metrics of input images using eagle algorithm.

\begin{tabular}{|c|c|c|c|}
\hline Inputs & Thickness & Mean & Standard Deviation \\
\hline 1 & 0.35503 & 0.04237 & 0.016560 \\
\hline 2 & 0.45060 & 0.04183 & 0.015588 \\
\hline 3 & 0.24184 & 0.04351 & 0.015193 \\
\hline 5 & 0.19432 & 0.04329 & 0.013595 \\
\hline 6 & 0.30043 & 0.04190 & 0.015678 \\
\hline 7 & 0.53720 & 0.04266 & 0.014890 \\
\hline 9 & 0.39266 & 0.04251 & 0.015023 \\
\hline 10 & 0.40385 & 0.04414 & 0.013999 \\
\hline
\end{tabular}

Source: own elaboration.

Thus, the eagle algorithm extracts the specific pattern to segment and concentrate on that pattern by clear segmentation with multi stochastic process and the better accuracy is achieved.

\section{CONCLUSIONS}

Our work, we proposed Eagle Algorithm (EA) for the enhancement of the segmentation process of knee images. The pre-processed image is a binary image with heavy noise. The noise is reduced by using Anisotropic Filter (AF). The brightness of the image is controlled and stabilized by Contrast Enhancement Method (CHE). The segmentation is done with two steps namely levy walk and intensive chasing. After the random search, the area is extracted accurately with intensive chasing. The algorithm fixes the cartilage part of the bone and segment with better clarity. The performance of the algorithm is analyzed using mat lab simulations. The thickness, mean and standard deviation result shows that the proposed method has developed result in contrast with the traditional methods. 


\section{REFERENCES}

Barthelemy, P., Bertolotti, J., \& Wiersma, D. S. (2008). A Lévy flight for light. Nature, 453(7194), 495-498. https://www.nature.com/articles/nature06948

Dogdas, B., Shattuck, D. W., \& Leahy, R. M. (2002). Segmentation of the skull in 3D human MR images using mathematical morphology. In Medical Imaging 2002: Image Processing (Vol. 4684, pp. 1553-1562). International Society for Optics and Photonics. https://ui.adsabs.harvard.edu/abs/2002SPIE.4684.1553D/abstract

Eckstein, F., Burstein, D., \& Link, T. M. (2006). Quantitative MRI of cartilage and bone: degenerative changes in osteoarthritis. $\mathcal{N M R}$ in Biomedicine: An International Fournal Devoted to the Development and Application of Magnetic Resonance In vivo, 19(7), 822854. https://www.researchgate.net/publication/6720453_Quantitative_MRI_of_ cartilage_and_bone_Degenerative_changes_in_osteoarthritis

Folkesson, J., Dam, E., Olsen, O. F., Pettersen, P., \& Christiansen, G. (2005). Automatic Segmentation of the Articular Cartilage in Knee MRI Using a Hierarchical Multi-class Classification Scheme. In Duncan, J.S., \& Gerig, G. (eds.) Medical Image Computing and Computer-Assisted Intervention - MICCAI 2005. Lecture Notes in Computer Science, vol 3749. Springer, Berlin, Heidelberg. https://doi. org/10.1007/11566465_41

Fripp, J., Grozier, S., Warfield, S. K., \& Ourselin, S. (2007). Automatic segmentation of the bone and extraction of the bone--cartilage interface from magnetic resonance images of the knee. Physics in Medicine \& Biology, 52(6), 1617. https://pubmed.ncbi. nlm.nih.gov/17327652/

Grau, V., Mewes, A. U. J., Alcaniz, M., Kikinis, R., \& Warfield, S. K. (2004). Improved watershed transform for medical image segmentation using prior information. IEEE transactions on medical imaging, 23(4), 447-458. https://ieeexplore. ieee.org/document/ 1281998

Kauffmann, G., Gravel, P., Godbout, B., Gravel, A., Beaudoin, G., Raynauld, J. P., ... \& de Guise, J. A. (2003). Computer-aided method for quantification of 
cartilage thickness and volume changes using MRI: validation study using a synthetic model. IEEE transactions on Biomedical Engineering, 50(8), 978-988. https: / / ieeexplore. ieee.org/document/1213850

Kubassova, O., Boyle, R. D., \& Pyatnizkiy, M. (2005). Bone segmentation in metacarpophalangeal MR data. In International Conference on Pattern Recognition and Image Analysis (pp. 726-735). Springer, Berlin, Heidelberg.

Li, L., Muneeswaran, V., Ramkumar, S., Emayavaramban, G., \& Gonzalez, G. R. (2019). Metaheuristic FIR filter with game theory based compression technique-A reliable medical image compression technique for online applications. Pattern Recognition Letters, 125, 7-12. https://doi.org/10.1016/j.patrec.2019.03.023

Liu, H., Yang, G., Pan, N., Song, E., \& Green, R. (2010). Denoising 3D MR images by the enhanced non-local means filter for Rician noise. Magnetic resonance imaging, 28(10), 1485-1496. https://doi.org/10.1016/j.mri.2010.06.023

Lorigo, L. M., Faugeras, O., Grimson, W. E. L., Keriven, R., \& Kikinis, R. (1998). Segmentation of bone in clinical knee MRI using texture-based geodesic active contours. In Wells W.M., Colchester, A., Delp, S. (eds.) Medical Image Computing and Computer-Assisted Intervention - MICGAI'98. Lecture Notes in Computer Science, vol 1496. Springer, Berlin, Heidelberg. https://doi.org/10.1007/BFb0056309

Lynch, J. A., Zaim, S., Zhao, J., Stork, A., Peterfy, G. G., \& Genant, H. K. (2000). Cartilage segmentation of 3D MRI scans of the osteoarthritic knee combining user knowledge and active contours. In Medical Imaging 2000: Image Processing (Vol. 3979, pp. 925-935). International Society for Optics and Photonics.

Muneeswaran, V., \& Rajasekaran, M.P. (2016). Analysis of particle swarm optimization based 2D FIR filter for reduction of additive and multiplicative noise in images. In International Conference on Theoretical Computer Science and Discrete Mathematics (pp. 165174). Springer, Cham.

Muneeswaran, V., \& Rajasekaran, M. P. (2016). Performance evaluation of radial basis function networks based on tree seed algorithm. In 2016 International Conference on 
Circuit, Power and Computing Technologies (ICCPCT) (pp. 1-4). IEEE. https: / / ieeexplore. ieee.org/document/7530267

Muneeswaran, V., \& Rajasekaran, M. P. (2017). Beltrami-Regularized Denoising Filter Based on Tree Seed Optimization Algorithm: An Ultrasound Image Application. In Satapathy, S., Joshi, A. (eds.) Information and Communication Technology for Intelligent Systems (ICTIS 2017) - Volume 1. ICTIS 2017. Smart Innovation, Systems and Technologies, vol 83. Springer, Cham. https://doi.org/10.1007/978-3-319-636733_54

Muneeswaran, V., \& Rajasekaran, M. P. (2018). Gallbladder shape estimation using tree-seed optimization tuned radial basis function network for assessment of acute cholecystitis. In Intelligent Engineering Informatics (pp. 229-239). Springer, Singapore.

Muneeswaran, V., \& Rajasekaran, M. P. (2019a). Automatic segmentation of gallbladder using bio-inspired algorithm based on a spider web construction model. The Fournal of Supercomputing, 75(6), 3158-3183. https://doi.org/10.1007/s11227017-2230-4

Muneeswaran, V., \& Rajasekaran, M. P. (2019b). Automatic segmentation of gallbladder using intuitionistic fuzzy based active contour model. In Microelectronics, Electromagnetics and Telecommunications (pp. 651-658). Springer, Singapore.

Muneeswaran, V., \& Rajasekaran, M. P. (2019c). Local contrast regularized contrast limited adaptive histogram equalization using tree seed algorithm - an aid for mammogram images enhancement. In Smart intelligent computing and applications (pp. 693-701). Springer, Singapore.

Norman, B., Pedoia, V., \& Majumdar, S. (2018). Use of 2D U-Net convolutional neural networks for automated cartilage and meniscus segmentation of knee MR imaging data to determine relaxometry and morphometry. Radiology, 288(1), 177185. https://pubmed.ncbi.nlm.nih.gov/29584598/

Pavlyukevich, I. (2007). Lévy flights, non-local search and simulated annealing. Fournal of Computational Physics, 226(2), 1830-1844. https://doi.org/10.1016/j.jcp.2007.06.008 
Pelletier, J. P., Raynauld, J. P., Abram, F., Haraoui, B., Choquette, D., \& MartelPelletier, J. (2008). A new non-invasive method to assess synovitis severity in relation to symptoms and cartilage volume loss in knee osteoarthritis patients using MRI. Osteoarthritis and cartilage, 16, S8-S13. https://pubmed.ncbi.nlm.nih.gov/18672386/

Peterfy, G. G., Schneider, E., \& Nevitt, M. (2008). The osteoarthritis initiative: report on the design rationale for the magnetic resonance imaging protocol for the knee. Osteoarthritis and cartilage, 16(12), 1433-1441. https://www.ncbi.nlm.nih.gov/pmc/ articles/PMC3048821/

Raynauld, J. P., Martel-Pelletier, J., Berthiaume, M.J., Labonté, F., Beaudoin, G., De Guise, J. A., ... \& Hochberg, M. G. (2004). Quantitative magnetic resonance imaging evaluation of knee osteoarthritis progression over two years and correlation with clinical symptoms and radiologic changes. Arthritis \& Rheumatism: Official fournal of the American College of Rheumatology, 50(2), 476-487. https://pubmed.ncbi.nlm.nih. gov/14872490/

Rini, G., Perumal, B., \& Rajasekaran, M. P. (2020). Automatic knee joint segmentation using Douglas-Rachford splitting method. Multimedia Tools and Applications, 79(9), 6599-6621. https://doi.org/10.1007/s1 1042-019-08303-8

Schmid, J., \& Magnenat-Thalmann, N. (2008). MRI bone segmentation using deformable models and shape priors. In International conference on medical image computing and computer-assisted intervention (pp. 119-126). Springer, Berlin, Heidelberg.

Stammberger, T., Eckstein, F., Michaelis, M., Englmeier, K. H., \& Reiser, M. (1999). Interobserver reproducibility of quantitative cartilage measurements: comparison of B-spline snakes and manual segmentation. Magnetic resonance imaging, 17(7), 1033-1042. https://pubmed.ncbi.nlm.nih.gov/10463654/

Tang, J., Millington, S., Acton, S. T., Grandall, J., \& Hurwitz, S. (2006). Surface extraction and thickness measurement of the articular cartilage from MR images using directional gradient vector flow snakes. IEEE Transactions on Biomedical Engineering, 53(5), 896-907. https://ieeexplore.ieee.org/document/1621141 
Wolf, M., Weierich, P., \& Niemann, H. (1997). Automatic Segmentation and 3D-Registration of a femoral bone in MR images of the knee. Pattern Recognition and Image Analysis (Advances in Mathematical Theory and Applications), 7(1), 152-165.

Yang, X. S., \& Deb, S. (2010). Eagle strategy using Lévy walk and firefly algorithms for stochastic optimization. In Nature Inspired Cooperative Strategies for Optimization (NICSO 2010) (pp. 101-111). Springer, Berlin, Heidelberg.

Yin, Y., Zhang, X., Williams, R., Wu, X., Anderson, D. D., \& Sonka, M. (2010). LOGISMOS - layered optimal graph image segmentation of multiple objects and surfaces: cartilage segmentation in the knee joint. IEEE transactions on medical imaging, 29(12), 2023-2037. https://pubmed.ncbi.nlm.nih.gov/20643602/ 